\title{
Influence of chronotype and social zeitgebers on sleep/wake patterns
}

\author{
A.L. Korczak¹, B.J. Martynhak¹, M. Pedrazzoli², A.F. Brito ${ }^{1}$ and F.M. Louzada1 \\ ${ }^{1}$ Setor de Ciências Biológicas, Departamento de Fisiologia, Universidade Federal do Paraná, Curitiba, \\ PR, Brasil \\ 2Departamento de Psicobiologia/Instituto de Sono, Universidade Federal de São Paulo, São Paulo, \\ SP, Brasil
}

Correspondence to: A.L. Korczak, Av. Francisco H. dos Santos, s/n, Setor de Ciências Biológicas, Departamento de Fisiologia, Centro Politécnico, UFPR, 81531-990 Curitiba, PR, Brasil

E-mail: annaligiak@yahoo.com.br

\begin{abstract}
Inter-individual differences in the phase of the endogenous circadian rhythms have been established. Individuals with early circadian phase are called morning types; those with late circadian phase are evening types. The Horne and Östberg Morningness-Eveningness Questionnaire (MEQ) is the most frequently used to assess individual chronotype. The distribution of MEQ scores is likely to be biased by several fact, ors, such as gender, age, genetic background, latitude, and social habits. The objective of the present study was to determine the effect of different social synchronizers on the sleep/wake cycle of persons with different chronotypes. Volunteers were selected from a total of 1232 UFPR undergraduate students who completed the MEQ. Thirty-two subjects completed the study, including 8 morning types, 8 evening types and 16 intermediate types. Sleep schedules were recorded by actigraphy for 1 week on two occasions: during the school term and during vacation. Sleep onset and offset times, sleep duration, and mid-sleep time for each chronotype group were compared by the Mann-Whitney U-test separately for school term and vacation. School term and vacation data were compared by the Wilcoxon matched-pair test. Morning types showed earlier sleep times and longer sleep duration compared with evening types (23:00 \pm 44 and $508.9 \pm 50.27$ vs 01:08 \pm 61.95 and $456.44 \pm 59.08$, for the weekdays during vacation). During vacation, the subjects showed later sleep times, except for the morning types, who did not exhibit differences for sleep onset times. The results support the idea that social schedules have an impact on the expression of circadian rhythmicity but this impact depends on the individual chronotype.
\end{abstract}

Key words: Chronotype; Sleep/wake cycle; Morning types; Evening types; Circadian rhythm; Social zeitgebers

Presented at the XI Congresso Brasileiro do Sono, Fortaleza, CE, Brazil, November 11-14, 2007.

Received November 8, 2007. Accepted August 29, 2008

\section{Introduction}

The human circadian timing system generates multiple circadian rhythms. One of the characteristics of a rhythm is the phase. The circadian phase can be defined as the time at which the circadian rhythm of a variable reaches a particular state (1). Substantial inter-individual differences in the circadian phase of several variables have been observed (2-7). Individuals with a relatively early circadian phase are morning types and those with a relatively late circadian phase are evening types. Morning and evening individuals differ in the phase of their endogenous circadian rhythms (1). The physiological markers of endogenous circadian rhythmicity are earlier in morning-type individuals than in evening types $(4,5,8,9)$. An individual's chronotype is usually evaluated by a questionnaire. The most widely used questionnaire is the Morningness-Eveningness Questionnaire (MEQ) of Horne and Östberg (10), although recently Roenneberg et al. (11) have proposed a new one, the Munich Chronotype Questionnaire (MCTQ). The MEQ scores are likely to be biased by several factors, such as age, latitude and social habits $(12,13)$. Moreover, several studies have also demonstrated that polymorphisms in clock genes might influence morningness-eveningness preference (14-17).

Studies have shown an association between sub- 
jects' season of birth and chronotype. Adan and Natale (18) reported that subjects born between April and September, containing summer, were significantly less likely to be morning types than subjects born between October and March, containing winter. This observation is consistent with the studies of Mongrain et al. (7). These investigators suggested that the association between season of birth and chronotype reflects the influence of the intensity of light and/or the variation in the photoperiod length during the gestational or perinatal period on the characteristics of the circadian system.

Morning types have earlier sleep schedules, wake up at an early hour and go to bed earlier compared with evening types $(7,11)$. Evening types are usually associated with a later bedtime and waking-up time, especially on weekends. Consequently, they have more irregular sleep/ wake habits. These subjects build up a sleep debt on workdays and extend their duration of sleep during the weekend $(11,19)$. Sleep debt is generated by the largest differences in sleep timing between work and free days. This disparity between social and biological time has been described as "social jetlag" (20). Apparently, evening-type individuals exhibit greater social jetlag than morning-type individuals. One alternative for analyzing the effect of social zeitgebers upon sleep/wake patterns is to compare the magnitude of the differences observed when subjects are affected by stronger or weaker social obligations.

Studies that describe the influence of chronotype upon sleep/wake patterns are often carried out with subjects under the influence of strong social zeitgebers, such as school and work schedules. Weekends are considered free days, which are compared with weekdays in order to estimate sleep-time discrepancies (19). However, it is well known that weekend sleep patterns usually involve a sleep rebound (11,19-22). As far as we know, studies considering vacation as free days instead of weekends, in order to reduce sleep rebound effects, have not been published. Comparisons between two situations, school term and vacation, would allow a better understanding of chronotype differences and the ability of circadian system to adjust to situations with distinct social constraints.

The aim of this study was to analyze the effect of changes in social synchronizers on the sleep/wake cycle of individuals with different chronotypes.

\section{Material and Methods}

\section{Subjects}

This study was approved by the Research and Ethics Committee of the Biological Sciences Institute of the Federal University of Paraná (UFPR). Subjects were selected by convenience sampling. The volunteers were UFPR undergraduate students and they signed an informed consent form before participating in the study. Volunteers lived in their own housing and were synchronized to have activities during the day and nocturnal rest, attending classes in the morning (8:00-12:00 am) and, eventually, in the afternoon (2:00-6:00 pm) from Monday through Friday during the school term. During vacation periods, they did not attend classes.

The volunteers answered a Portuguese version of the Horne-Östberg questionnaire (13) for determination of diurnal preference.

Volunteers were selected from a database of 1232 subjects, 499 men and 733 women, mean age 20.5 years, who answered the MEQ. A total of 32 subjects completed the study, including 8 morning types ( 7 women and 1 man), 8 evening types ( 6 women and 2 men) and 16 intermediate types (12 women and 4 men). The mean age was $19.62 \pm$ 1.85 years. None of the volunteers had a diagnosed sleep disturbance. No subject underwent medical treatment immediately before or during this study.

The volunteers completed a sleep log and wore an activity monitor (Mini-Motionlogger, Zero crossing mode, Ambulatory Monitoring Inc., USA) on their non-dominant wrist for 7 consecutive days ( 5 weekdays and 2 weekend days) during the school term and another 7 consecutive days during vacation to record activity levels at 1-min intervals. The actigraph data were analyzed by the ActionW software program for measures of sleep onset time, sleep offset time, and the duration of nocturnal sleep. The bedtime and wake-up time noted in the subject's sleep log were used to guide analysis of the actigraph data recordings (23).

The following dependent variables were obtained for each individual: sleep onset time, sleep offset time, midsleep time and night-sleep duration. Three means were obtained for each dependent variable: entire week, weekdays and weekends. The independent variables were the chronotype and the two situations related to social synchronizers: school term and vacation. The dependent variables for each chronotype group were compared by means of the Mann-Whitney U-test, applied separately for school term and vacation. The school term and vacation data were compared for each chronotype using the Wilcoxon matched-pair test. $\mathrm{P}<0.05$ was considered to be significant.

\section{Results}

Entire week ( 7 days)

The sleep variables for each chronotype and social schedules for the entire week are plotted in Figure $1 \mathrm{~A}$. 
There were differences between the chronotypes, both during the school term and during vacation (Mann-Whitney U-test). Morning types had statistically significant $(\mathrm{P}<$ 0.05 ) earlier sleep, wake-up times and mid-sleep times than intermediate types for the school term and for vacation and also compared with evening types $(P<0.05)$. Intermediate types had significantly $(P<0.05)$ earlier sleep, wake-up times and mid-sleep times than evening types for the school term and for vacation. The sleep duration of the morning group was statistically $(P<0.05)$ longer than the intermediate and evening types for the school term and for vacation.

Sleep onset for evening types and intermediate types was significantly later during vacation than during the school term $(P<0.05)$. This delay was not observed in morning-type individuals. Morning, evening and intermediate types woke up significantly later $(P<0.05)$ during vacation and their mid-sleep times were also later $(P<$ $0.05)$ during vacation than during the school term. There was also a significant increase in night-sleep duration during vacation $(P<0.05$; Table 1$)$.

\section{Weekdays (5 days)}

The sleep variables for each chronotype and social schedule for the weekdays are plotted in Figure 1B. The Mann-Whitney U-test detected significant $(P<0.05)$ differences between morning and evening types for the school term and for vacation and between morning and intermediate types for the school term and for vacation for all dependent variables, with the exception of offset time during the school term. When evening and intermediate types were compared during vacation, only sleep onset time was significantly different. As previously observed for the entire week, the morning-type group slept more than the evening and intermediate types, both during the school term and during vacation $(P<0.05)$.

During weekdays, sleep onset time was significantly $(P$ $<0.05$ ) later in evening and intermediate types during vacation compared to during the school term. This was not observed in morning-type individuals. As observed in the entire week analysis, morning, evening and intermediate individuals had significantly later wake-up and mid-sleep times during vacation than during the school term and had a significant increase in their night-sleep duration during vacation $(P<0.05$; Table 1$)$.

\section{Weekends (2 days)}

The sleep variables for each chronotype and social schedules for the weekends are plotted in Figure 1C. The Mann-Whitney U-test detected differences between all chronotype groups for onset, offset and mid-sleep times
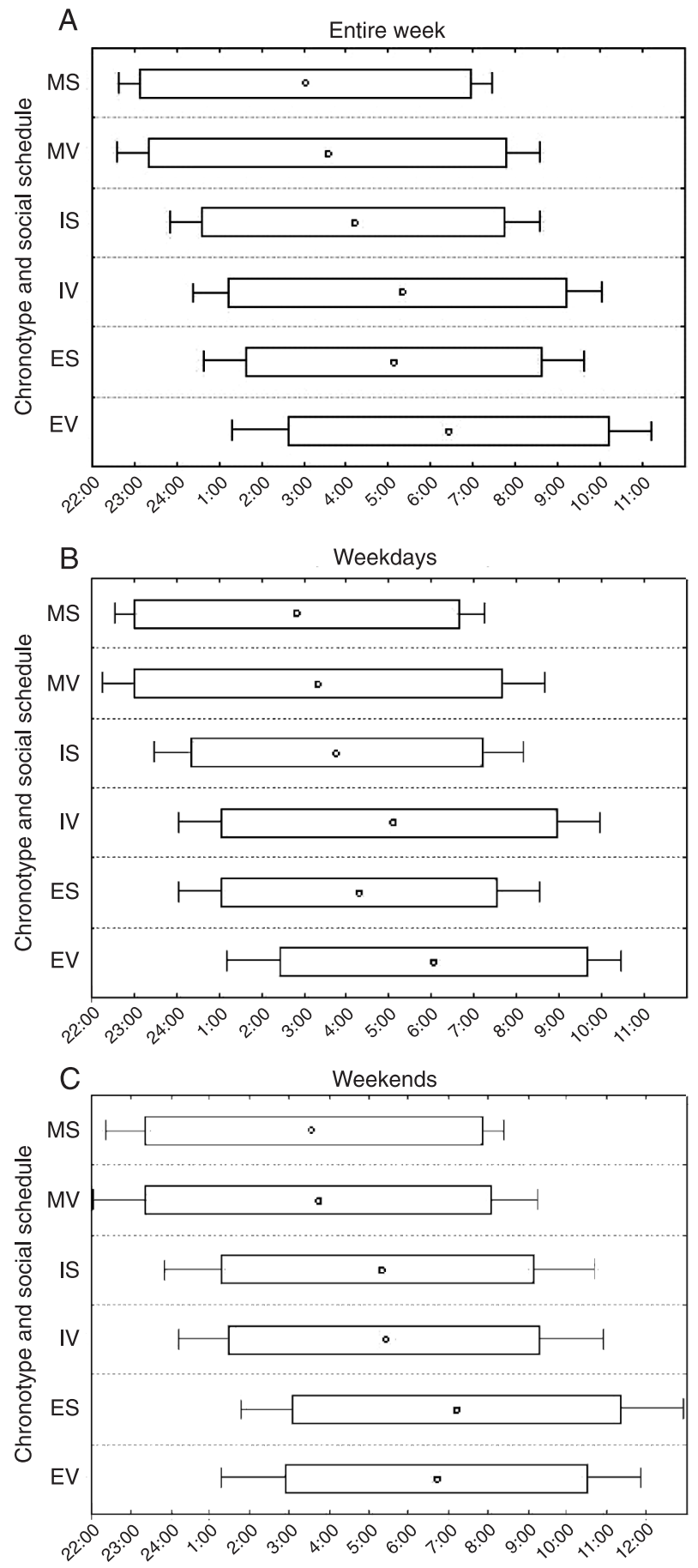

Figure 1. Sleep patterns of university students as a function of chronotype and activity. $A$, Entire week; $B$, weekdays; $C$, weekends. The sleep patterns are represented by horizontal bars. The first letter for each line on the $y$-axis indicates the chronotype ( $\mathrm{M}=$ morning, $\mathrm{I}=$ intermediate, and $\mathrm{E}$ = evening types); the second letter represents the social schedule $(S=$ school term and $\mathrm{V}=$ vacation). The circle in the center of the bar is the midsleep time. The standard deviations for sleep onset and offset are indicated at the left and right of the bars, respectively. 
during the school term and during vacation, with the exception of offset time during vacation, when comparing morning and intermediate types. Morning types slept earlier, woke up earlier and had an earlier mid-sleep time than intermediate types for the school term and for vacation, and evening types for the school term and for vacation. Intermediate types had earlier sleep, wake-up times, just during the school term, and mid-sleep times than evening types for the school term and for vacation. No significant differences were found between chronotypes when night-sleep duration was analyzed (Table 1).

The Wilcoxon matched-pair test did not detect any significant differences in sleep patterns on weekends comparing the school term and vacation data.

\section{Discussion}

This study analyzed the sleep/wake cycle of individuals affected by different social synchronizers. It is well known that social obligations and proximate social cues directly influence sleep and wake times (20). It is not possible to determine whether social stimuli affect circadian timing independent of their role in regulating light exposure (24).

This is the first study showing objective sleep/wake data in a situation with weak social constraints and in the absence of sleep rebound effects, which were observed on weekends. Our results suggest that under these conditions, individual differences in sleep needs and sleep timing can be better depicted.

The results of this study showed that, as expected, when exposed to stronger or weaker social obligations, morning-type individuals had earlier sleep onset and offset times and evening types had significantly later sleep onset and offset times, as described elsewhere $(6,10,11,19,21$, 22,24-28).

We observed several differences in sleep patterns during the school term and during vacation. All chrono-

Table 1. Sleep patterns during the school term and vacation.

\begin{tabular}{|c|c|c|c|}
\hline & Morning types $(\mathrm{N}=8)$ & Intermediate types $(\mathrm{N}=16)$ & Evening types $(\mathrm{N}=8)$ \\
\hline \multicolumn{4}{|l|}{ School term } \\
\hline Sleep onset (entire week) & $23: 06 \pm 24.8^{\mathrm{I}, \mathrm{E}}$ & $00: 34 \pm 45.2^{*, \mathrm{M}, \mathrm{E}}$ & $01: 37 \pm 57.8^{*, M, I}$ \\
\hline Sleep onset (weekdays) & $23: 00 \pm 27.0^{\mathrm{I}, \mathrm{E}}$ & $00: 17 \pm 43.0^{*, M}$ & $00: 27 \pm 119.6^{\star, M}$ \\
\hline Sleep onset (weekends) & $23: 22 \pm 55.4^{\mathrm{I}, \mathrm{E}}$ & $01: 20 \pm 89.6^{\mathrm{M}, \mathrm{E}}$ & $03: 03 \pm 74.0^{\mathrm{M}, \mathrm{I}}$ \\
\hline Sleep offset (entire week) & $06: 56 \pm 32.5^{\star, I, E}$ & $07: 46 \pm 53.4^{*, M, E}$ & $08: 35 \pm 57.2^{*, \mathrm{M}, \mathrm{I}}$ \\
\hline Sleep offset (weekdays) & $06: 40 \pm 34.1^{*}$ & $07: 13 \pm 55.1^{*}$ & $07: 33 \pm 60.2^{*}$ \\
\hline Sleep offset (weekends) & $07: 51 \pm 31.3^{\mathrm{I}, \mathrm{E}}$ & $09: 16 \pm 93.6^{\mathrm{M}, \mathrm{E}}$ & $11: 20 \pm 106.7^{\mathrm{M}, \mathrm{I}}$ \\
\hline Sleep duration (entire week) & $459.86 \pm 20.3^{\mathrm{l}, \mathrm{E}}$ & $419.08 \pm 53.0^{\mathrm{M}}$ & $406.82 \pm 33.1^{\mathrm{M}}$ \\
\hline Sleep duration (weekdays) & $447.05 \pm 21.9^{\mathrm{l}, \mathrm{E}}$ & $404.75 \pm 60.2^{\mathrm{M}}$ & $379.05 \pm 47.7^{\mathrm{M}}$ \\
\hline Sleep duration (weekends) & $491.88 \pm 42.8$ & $454.91 \pm 82.2$ & $468.06 \pm 76.1$ \\
\hline Mid-sleep (entire week) & $03: 02 \pm 28.4^{*}, \mathrm{I}, \mathrm{E}$ & $04: 12 \pm 42.5^{\star}, \mathrm{M}, \mathrm{E}$ & $05: 08 \pm 56.3^{*, M, I}$ \\
\hline Mid-sleep (weekdays) & $02: 50 \pm 29.5^{\star}, \mathrm{I}, \mathrm{E}$ & $03: 45 \pm 40.2^{*, M}$ & $04: 18 \pm 55.3^{*}, M$ \\
\hline Mid-sleep (weekends) & $03: 33 \pm 41.0^{\mathrm{l}, \mathrm{E}}$ & $05: 18 \pm 80.2^{\mathrm{M}, \mathrm{E}}$ & $07: 12 \pm 75.7^{M}, 1$ \\
\hline \multicolumn{4}{|l|}{ Vacation } \\
\hline Sleep onset (entire week) & $23: 19 \pm 43.6^{\mathrm{I}, \mathrm{E}}$ & $01: 18 \pm 59.3^{*}, \mathrm{M}, \mathrm{E}$ & $02: 37 \pm 79.6^{*, M}, \mathrm{I}$ \\
\hline Sleep onset (weekdays) & $23: 00 \pm 44.0^{\mathrm{l}, \mathrm{E}}$ & $01: 08 \pm 62.0^{*, M, E}$ & $02: 27 \pm 76.5^{*, M, I}$ \\
\hline Sleep onset (weekends) & $23: 20 \pm 78.9^{\mathrm{I}, \mathrm{E}}$ & $01: 31 \pm 80.5^{\mathrm{M}, \mathrm{E}}$ & $02: 54 \pm 91.2^{\mathrm{M}, \mathrm{I}}$ \\
\hline Sleep offset (entire week) & $07: 48 \pm 51.2^{*, \mathrm{I}, \mathrm{E}}$ & $09: 20 \pm 62.8^{*, M, E}$ & $10: 16 \pm 61.2^{*, M, I}$ \\
\hline Sleep offset (weekdays) & $07: 39 \pm 61.7^{*, I, E}$ & $09: 05 \pm 70.6^{*}, \mathrm{M}$ & $09: 40 \pm 48.0 *, M$ \\
\hline Sleep offset (weekends) & $08: 07 \pm 62.7 \mathrm{E}$ & $09: 19 \pm 94.6$ & $10: 32 \pm 74.5^{\mathrm{M}}$ \\
\hline Sleep duration (entire week) & $497.73 \pm 42.4^{*, \mathrm{I}, \mathrm{E}}$ & $458.25 \pm 39.7^{*, M}$ & $440.8 \pm 33.2^{*, M}$ \\
\hline Sleep duration (weekdays) & $508.9 \pm 50.3^{*, \mathrm{I}, \mathrm{E}}$ & $456.44 \pm 59.1^{*}, \mathrm{M}$ & $420.9 \pm 47.3^{*}, \mathrm{M}$ \\
\hline Sleep duration (weekends) & $501.38 \pm 76.3$ & $462.03 \pm 101.5$ & $465.75 \pm 64.4$ \\
\hline Mid-sleep (entire week) & $03: 34 \pm 44.5^{\star, I, E}$ & $05: 19 \pm 58.9^{*}, \mathrm{M}, \mathrm{E}$ & $06: 25 \pm 67.5^{\star}, \mathrm{M}, \mathrm{I}$ \\
\hline Mid-sleep (weekdays) & $03: 20 \pm 48.0^{*}, \mathrm{I}, \mathrm{E}$ & $05: 07 \pm 61.8^{*, M}$ & $06: 03 \pm 59.5^{\star, M}$ \\
\hline Mid-sleep (weekends) & $03: 45 \pm 59.0^{1, E}$ & $05: 25 \pm 76.7^{\mathrm{M}, \mathrm{E}}$ & $06: 43 \pm 76.6^{\mathrm{M}, \mathrm{I}}$ \\
\hline
\end{tabular}

Data are reported as mean \pm SD. Sleep onset, offset and mid-sleep times are reported as time with SD in minutes. Sleep duration is reported in minutes.

${ }^{*} \mathrm{P}<0.05$, school term vs vacation in the same chronotype (Wilcoxon matched-pair test). The chronotype letters $\mathrm{M}$ (morning type), I (intermediate type) and $E$ (evening type) indicate significant differences $(P<0.05)$ between chronotype groups in the same schedule (school term or vacation) (Mann-Whitney U-test). 
type groups had delayed sleep offset and mid-sleep times and an increase in sleep duration during vacation. Evening-type individuals exhibit a greater misalignment between their sleep preferences and social schedules and could eventually suffer "social jetlag" (20). These differences between the school term and vacation suggest that their adjustment to social schedules requires a greater phase advance of the sleep/wake cycle, which would occur only partially, with consequent partial sleep deprivation.

Interestingly, the morning group slept longer than evening-type individuals, both during the school term and the vacation period. This result was not observed in previous studies. On the contrary, previous studies $(19,29,30)$ found that evening types slept longer than morning types, in accordance with studies that showed a faster decrease of slow-wave sleep in morning-type individuals (31). It is well known that evening-type individuals build up a sleep debt during the week and extend their duration of sleep during the weekend (19). This sleep debt could be the reason for the association between evening types and a greater need for sleep. According to this view, one possible interpretation of our data is that, during vacation, when sleep duration supposedly meets sleep needs, evening types appear to continue to be affected by social constraints. One alternative view, which is not supported by previous studies (6), is that morning types have greater sleep needs compared with evening types.

Unfortunately, our society largely ignores these important differences in circadian phase between individuals (32). Detailed knowledge of how the interaction between the circadian system and social zeitgebers shape chronotype is extremely relevant for determining school times and shift workers' schedules (33).

\section{References}

1. Van Dongen HP. Inter and intra-individual differences in circadian phase. Leiden: Leiden University Press; 1998.

2. Foret J, Benoit O, Royant-Parola S. Sleep schedules and peak times of oral temperature and alertness in morning and evening 'types'. Ergonomics 1982; 25: 821-827.

3. Kerkhof GA, Van Dongen HP. Morning-type and eveningtype individuals differ in the phase position of their endogenous circadian oscillator. Neurosci Lett 1996; 218: 153-156.

4. Duffy JF, Dijk DJ, Hall EF, Czeisler CA. Relationship of endogenous circadian melatonin and temperature rhythms to self-reported preference for morning or evening activity in young and older people. J Investig Med 1999; 47: 141-150.

5. Baehr EK, Revelle W, Eastman Cl. Individual differences in the phase and amplitude of the human circadian temperature rhythm: with an emphasis on morningness-eveningness. J Sleep Res 2000; 9: 117-127.

6. Mongrain V, Lavoie S, Selmaoui B, Paquet J, Dumont M. Phase relationships between sleep-wake cycle and underlying circadian rhythms in morningness-eveningness. J Biol Rhythms 2004; 19: 248-257.

7. Mongrain V, Paquet J, Dumont M. Contribution of the photoperiod at birth to the association between season of birth and diurnal preference. Neurosci Lett 2006; 406: 113-116.

8. Kerkhof GA. Inter-individual differences in the human circadian system: a review. Biol Psychol 1985; 20: 83-112.

9. Bailey SL, Heitkemper MM. Circadian rhythmicity of cortisol and body temperature: morningness-eveningness effects. Chronobiol Int 2001; 18: 249-261.

10. Horne JA, Ostberg $\mathrm{O}$. A self-assessment questionnaire to determine morningness-eveningness in human circadian rhythms. Int J Chronobiol 1976; 4: 97-110.

11. Roenneberg T, Wirz-Justice A, Merrow M. Life between clocks: daily temporal patterns of human chronotypes. $J$ Biol Rhythms 2003; 18: 80-90.

12. Smith CS, Folkard S, Schmieder RA, Parra LF, Spelten E,
Almiral $\mathrm{H}$, et al. Investigation of morning-evening orientation in six countries using the preferences scale. Pers Individ Dif 2002; 32: 949-968.

13. Benedito-Silva AA, Menna-Barreto L, Cipolla-Neto J, Marques $\mathrm{N}$, Tenreiro $\mathrm{S}$. Latitude and social habits as determinants of the distribution of morning and evening types in Brazil. Bio Rhythm Res 1998; 29: 591-597.

14. Katzenberg D, Young T, Finn L, Lin L, King DP, Takahashi JS, et al. A CLOCK polymorphism associated with human diurnal preference. Sleep 1998; 21: 569-576.

15. Archer SN, Robilliard DL, Skene DJ, Smits M, Williams A, Arendt $\mathrm{J}$, et al. A length polymorphism in the circadian clock gene Per3 is linked to delayed sleep phase syndrome and extreme diurnal preference. Sleep 2003; 26: 413-415.

16. Pereira DS, Tufik S, Louzada FM, Benedito-Silva AA, Lopez $A R$, Lemos NA, et al. Association of the length polymorphism in the human Per3 gene with the delayed sleepphase syndrome: does latitude have an influence upon it? Sleep 2005; 28: 29-32.

17. Carpen JD, von Schantz M, Smits M, Skene DJ, Archer SN. A silent polymorphism in the PER1 gene associates with extreme diurnal preference in humans. J Hum Genet 2006; 51: $1122-1125$.

18. Adan A, Natale V. Gender differences in morningnesseveningness preference. Chronobiol Int 2002; 19: 709-720.

19. Taillard J, Philip P, Bioulac B. Morningness/eveningness and the need for sleep. J Sleep Res 1999; 8: 291-295.

20. Wittmann M, Dinich J, Merrow M, Roenneberg T. Social jetlag: misalignment of biological and social time. Chronobiol Int 2006; 23: 497-509.

21. Giannotti F, Cortesi F, Sebastiani T, Ottaviano S. Circadian preference, sleep and daytime behaviour in adolescence. $J$ Sleep Res 2002; 11: 191-199.

22. Roenneberg T, Kuehnle T, Pramstaller PP, Ricken J, Havel M, Guth A, et al. A marker for the end of adolescence. Curr 
Biol 2004; 14: R1038-R1039.

23. Acebo C, Sadeh A, Seifer R, Tzischinsky O, Wolfson AR, Hafer A, et al. Estimating sleep patterns with activity monitoring in children and adolescents: how many nights are necessary for reliable measures? Sleep 1999; 22: 95-103. Erratum in: Sleep 22: 143.

24. Mistlberger RE, Skene DJ. Social influences on mammalian circadian rhythms: animal and human studies. Biol Rev Camb Philos Soc 2004; 79: 533-556.

25. Vink JM, Groot AS, Kerkhof GA, Boomsma DI. Genetic analysis of morningness and eveningness. Chronobiol Int 2001; 18: 809-822.

26. Monk TH, Buysse DJ, Welsh DK, Kennedy KS, Rose LR. A sleep diary and questionnaire study of naturally short sleepers. J Sleep Res 2001; 10: 173-179.

27. Lima PF, Medeiros AL, Araujo JF. Sleep-wake pattern of medical students: early versus late class starting time. Braz $J$
Med Biol Res 2002; 35: 1373-1377.

28. Taillard J, Philip P, Coste O, Sagaspe P, Bioulac B. The circadian and homeostatic modulation of sleep pressure during wakefulness differs between morning and evening chronotypes. J Sleep Res 2003; 12: 275-282.

29. Ong JC, Huang JS, Kuo TF, Manber R. Characteristics of insomniacs with self-reported morning and evening chronotypes. J Clin Sleep Med 2007; 3: 289-294.

30. Carrier J, Monk TH, Buysse DJ, Kupfer DJ. Sleep and morningness-eveningness in the 'middle' years of life (2059 y). J Sleep Res 1997; 6: 230-237.

31. Mongrain V, Carrier J, Dumont M. Chronotype and sex effects on sleep architecture and quantitative sleep EEG in healthy young adults. Sleep 2005; 28: 819-827.

32. Anonymous. Timing is everything. Nature 2003; 425: 885.

33. Roenneberg T, Merrow M. Circadian clocks - the fall and rise of physiology. Nat Rev Mol Cell Biol 2005; 6: 965-971. 